\title{
HIGHLIGHTS
}

CANCER

\section{Long-term use of metformin could protect against breast cancer}

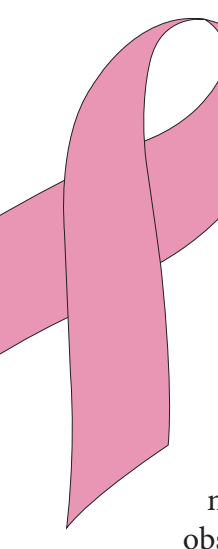

Long-term treatment with metformin reduces the relative risk estimate of incident breast cancer in patients with type 2 diabetes mellitus, suggest results of a recent study published in Diabetes Care.

Over the past few years, data from several studies indicated that metformin exhibited antitumor effects in vitro and in animal models. In 2005, researchers of an observational study reported a lower frequency of cancer in patients who were treated with metformin compared with those not receiving this drug. The study results, however, had not been stratified for individual cancer types. "These results made our group curious whether metformin may be associated with an altered risk of breast cancer in humans," says Michael Bodmer from the University Hospital Basel, Switzerland. "Therefore, since we work with the UK-based General Practice Research Database (GPRD), we decided to perform a nested case-control analysis".

Bodmer and colleagues used the GPRD database to determine the incidence of breast cancer in 22,621 women with type 2 diabetes mellitus (aged 30-79 years) who were treated with oral antidiabetic drugs, including sulfonylureas, biguanides, thiazolidinediones, $\alpha$-glucosidase inhibitors or prandial glucose regulators, with or without concomitant insulin use. Patients who were treated with insulin monotherapy were not included.

The investigators identified 305 cases with a recorded incident diagnosis of breast cancer (mean age 67.5 years) and matched these to 1,153 cancer-free women by age, sex and calendar time (that is, the date of cancer diagnosis in order to compare drug exposure at the same point in time). Bodmer et al. also adjusted the analyses for important risk factors of breast cancer, such as BMI and estrogen use, and stratified the results for glycemic control $\left(\mathrm{HbA}_{1 \mathrm{c}}\right)$ and diabetes duration.

"Our most notable finding was a decreased relative risk estimate of incident breast cancer in patients using metformin on a long-term basis (approximately 5 years) compared to patients with diabetes mellitus who were not treated with this drug," summarizes Bodmer. "We did not find any alteration of breast cancer risk in relation to short-term use of metformin or any other use of another oral antidiabetic drug".

So far, available evidence from several studies that link metformin use to a decreased risk of overall cancer-related mortality is in accordance with the findings by Bodmer and co-workers.

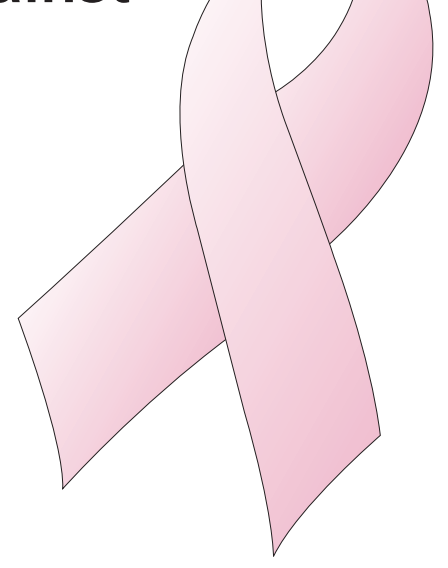

"We are now waiting for the results of ongoing clinical trials investigating the potential role of metformin as an add-on therapy to standard chemotherapy," comments Bodmer further.

Whether or not metformin is indeed beneficial as a prophylactic agent against breast cancer will require further studies. However, as these studies will need to include a large number of participants over a long period of time-Bodmer's results suggest years of treatment-such trials will prove difficult to accomplish.

"Observational evidence, including our results, does not prove causality," concedes Bodmer. "Therefore, no immediate change in breast cancer treatment is justified at this point".

\section{Linda Koch}

Original article Bodmer, M. et al. Long-term metformin use is associated with decreased risk of breast cancer. Diabetes Care 33, 1304-1308 (2010) 